\title{
Stability of Supersonic Boundary Layers on a Cone at an Angle of Attack
}

\author{
P.Balakumar \\ Flow Physics and Control Branch \\ NASA Langley Research Center, Hampton, VA 23681
}

\begin{abstract}
The stability and receptivity of three-dimensional supersonic boundary layers over a $7^{\circ}$ sharp tipped straight cone at an angle of attack of $4.2^{\circ}$ is numerically investigated at a free stream Mach number of 3.5 and at two high Reynolds numbers, 0.25 and $0.50^{*} 10^{6} /$ inch. The generation and evolution of stationary crossflow vortices are also investigated by performing simulations with three-dimensional roughness elements located on the surface of the cone. The flow fields with and without the roughness elements are obtained by solving the full Navier-Stokes equations in cylindrical coordinates using the fifth-order accurate weighted essentially non-oscillatory (WENO) scheme for spatial discretization and using the thirdorder total-variation-diminishing (TVD) Runge-Kutta scheme for temporal integration. Stability computations reveal that the azimuthal wavenumbers are in the range of $m \sim 25-50$ for the most amplified traveling disturbances and in the range of $m \sim 40-70$ for the stationary disturbances. The $\mathbf{N}$-Factor computations predicted that transition would occur further forward in the middle of the cone compared to the transition fronts near the windward and the leeward planes. The simulations revealed that the crossflow vortices originating from the nose region propagate towards the leeward plane. No perturbations were observed in the lower part of the cone.
\end{abstract}

\section{Introduction}

Three-dimensional boundary layers exist when the inviscid streamlines are curved in the spanwise direction. When the inviscid streamlines are curved, there exists a pressure gradient in the direction normal to the inviscid streamlines. Inside the boundary layer, due to the viscous effect, the velocity is smaller than that in the inviscid region. Hence, this pressure gradient causes a velocity component, called crossflow velocity, inside the boundary layer that is perpendicular to the inviscid-velocity vector. This crossflow velocity contains an inflection point in its profile and causes a new instability called crossflow instability. The crossflow instability is unstable to three-dimensional traveling and stationary disturbances. The stationary disturbances originate from isolated roughness elements and appear as corotating vortices. The stationary crossflow vortices dominate the transition process in most of the cases except in high turbulence environments. This phenomenon is observed in several incompressible and compressible flows including swept wings, rotating disks, rotating cones and cones at angles of attack. The linear and nonlinear crossflow instability in incompressible flow is well explained by the pioneering work of Gergory et al. (1955), Dehyle and Bippes (1996), Saric et al. (1998) and Malik et al. (1994). The major findings about the linear and nonlinear crossflow instability in incompressible flows are summarized in a review paper by Saric et al. (2003).

Early investigations of the stability characteristics of supersonic boundary layers ${ }^{6-8}$ revealed the important finding that the unstable disturbances in supersonic boundary layers are three-dimensional. They also found that the wave angles of the most amplified disturbances are inclined around 60-65 degrees from the inviscid streamlines in a boundary layer with an edge Mach number of 3.5. The linear instability of axi-symmetric three-dimensional compressible boundary layers for a rotating cone was numerically investigated by Balakumar and Reed (1991). Their calculations showed that the growth rate of the traveling disturbances is increased by a factor of 2 to 4 due to the presence of the crossflow compared with the two-dimensional flow over a non-rotating cone and this increase decreases with increasing Mach number. 
There have been several experiments performed to measure the transition front in compressible threedimensional boundary layers and a partial list of these is provided in Malik and Balakumar (1992). Most of the experiments were performed in conventional tunnels except for those performed in NASA Langley's Supersonic Low Disturbance Tunnel (Chen et al. 1988, King 1992, and Cattafesta et al. 1995). Chen et al. (1988) measured the transition onset on a $5^{\circ}$ half-angle cone at zero angle of attack using thermocouples, Cattafesta et al. (1995) investigated the transition onset on a swept wing using temperature sensitive paint. King (1992) measured the transition on a $5^{\circ}$ half-angle sharp cone at several angles of attack using Preston tubes. The linear stability and transition prediction calculations for King's (1992) experiment were performed in Refs. (10), (14), and (15). The stability characteristics of flows over cones at angles of attack can be divided into three parts. One is close to the windward symmetry plane, where the crossflow is weak and the transition occurs due to the unstable first modes. The second is the region near the leeward plane, where the crossflow is again weak but the velocity profiles become highly inflectional due to the accumulation of low speed fluid from either side of the symmetry plane. This region becomes much more unstable and the transition occurs much earlier than that along the windward plane. Third is the larger middle region, where the crossflow is strong and the transition occurs due to traveling and/or stationary crossflow disturbances. The crossflow gradually increases from the windward plane with increasing azimuthal angle, reaches its peak around $120^{\circ}$ and decreases again towards the leeward plane. This reflects in the computed transition fronts as the transition front moves upstream with increasing azimuthal angle up to $120^{\circ}$ and moves downstream again with further increase in the azimuthal angle. However, in the King $^{12}$ experiment, at high angles of attack, the transition front continuously moved upstream up to the leeward plane. A possible reason for the discrepancy between the predicted behavior of the transition front and the results of King will be discussed in the results section. Supersonic flow over a cone at an angle of attack is generally used as a generic model to study the crossflow instability in three-dimensional supersonic boundary layers. However, it has to be noted that this boundary layer exhibits different stability characteristics in different parts of the domain. This is the difference between the flow over an infinite swept wing and the flow over a cone at an angle of attack. In the former, the mean flow field and the stability properties are uniform in the spanwise direction.

There are not many detailed stability and transition experiments performed in three-dimensional supersonic boundary layers. Our objective is to investigate numerically the linear instability, transition onset, receptivity, and the growth of stationary crossflow vortices in supersonic boundary layers over a sharp cone at an angle of attack. We consider a supersonic flow of $M=3.5$ over a $7^{\circ}$ half-angle sharp cone at an angle of attack of $4.2^{\circ}$ (Fig. 1). The nose radius is 0.001 inches. These parameters are selected to validate and support the stability and flow control experiments that are being performed in NASA Langley's Supersonic Low Disturbance Tunnel. The simulations are performed by solving the threedimensional Navier-Stokes equations in cylindrical coordinates using the fifth-order accurate weighted essentially non-oscillatory (WENO) scheme for spatial discretization and using the third-order totalvariation-diminishing (TVD) Runge-Kutta scheme for temporal integration. The stability computations are performed using the local parallel theory and the N-Factor computations are performed using the three-dimensional transition prediction code emalik ${ }^{3 d}{ }^{16}$. Receptivity analysis is carried out by performing simulations with three-dimensional roughness elements placed around the cone at a fixed distance from the apex of the cone. Computations are performed for unit Reynolds numbers of 0.25 and $0.50 * 10^{6} /$ inch. Most of the results will be presented for the lower unit Reynolds number case. The governing equations and a brief note about the solution procedure are discussed in section II. Mean flow profiles, linear stability results, transition onsets, receptivity and the evolution of crossflow vortices originating from the roughness elements are presented in section III. Conclusions are drawn in section IV.

\section{Governing Equations}

The equations solved are the three-dimensional unsteady compressible Navier-Stokes equations in conservation form in cylindrical coordinates 


$$
\frac{\partial}{\partial t} r Q+\frac{\partial r F}{\partial x}+\frac{\partial r G}{\partial r}+\frac{\partial H}{\partial \theta}=S
$$

$$
Q=\left|\begin{array}{l}
\rho \\
\rho u \\
\rho v \\
\rho w \\
\rho E
\end{array}\right|
$$

Here $(x, r, \theta)$ are the cylindrical coordinates, $(u, v, w)$ are the velocity components, $\rho$ is the density, and $p$ is the pressure. $E$ is the total energy given by

$$
\begin{aligned}
& E=e+\frac{u^{2}+v^{2}+w^{2}}{2}, \\
& e=c_{v} T, p=\rho R T .
\end{aligned}
$$

Here $e$ is the internal energy and $T$ is the temperature. The fluxes $F, G, H$, and the source term $S$ are described in Ref. 17. The viscosity $(\mu)$ is computed using Sutherland's law and the coefficient of conductivity $(k)$ is given in terms of the Prandtl number $\operatorname{Pr}$. The variables $\rho, p, T$ and velocity are nondimensionalized by their corresponding reference variables $\rho_{\alpha}, p_{\alpha}, T_{\infty}$ and $\sqrt{R T_{\infty}}$, respectively. The reference value for length is computed by $\sqrt{v x_{0} / U_{\infty}}$, where $x_{0}$ is a reference location. For the computation, the equations are transformed from the physical coordinate system $(x, r, \theta)$ to the computational curvilinear coordinate system $(\xi, \eta, \zeta)$ in a conservative manner.

\section{A. Solution Algorithm}

The governing equations are solved using a fifth order accurate weighted essentially non-oscillatory (WENO) scheme for space discretization and using a third order, total variation diminishing (TVD) Runge-Kutta scheme for time integration. These methods are suitable in flows with discontinuities or high gradient regions. The governing equations are solved discretely in a uniform structured computational domain where flow properties are known point wise at the grid nodes. In a given direction, the spatial derivatives are approximated to a higher order at the nodes, using the neighboring nodal values in that direction. The resulting equations are then integrated in time to get the point values as a function of time. Since the spatial derivatives are independent of the coordinate directions, multi dimensions can be easily added to the method. It is well known that approximating a discontinuous function by a higher-order (two or more) polynomial generally introduces oscillatory behavior near the discontinuity, and this oscillation increases with the order of the approximation. The essentially non-oscillatory (ENO) method and its successor the WENO method were developed to maintain the higher-order approximations in the smooth regions and to eliminate or suppress the oscillatory behavior near the discontinuities. These methods systematically adopt or select the stencils based on the smoothness of the function that is being approximated. Reference 18 explains the WENO and the TVD methods and the formulas. Reference 19 gives the application of the ENO method to the N-S equations. Reference 20 describes in detail the solution method implemented in this computation. 
At the outflow boundary, characteristic boundary conditions are used. At the wall, viscous conditions are used for the velocities and a constant temperature condition is employed for the temperature. The density at the wall is computed from the continuity equation. In the azimuthal direction, symmetric conditions are imposed at the $\theta=0^{\circ}$ and $\theta=180^{\circ}$ boundaries. The free-stream values are prescribed at the upper boundary that lies outside the bow shock. The steady state computations are performed using a variable time step until the maximum residual reaches a small value $\sim 10^{-10}$.

\section{Results}

The computations are performed for a supersonic flow with a Mach number of $M=3.5$ over a 7degree half-angle cone at an angle of attack of 4.2 degrees. The length of the cone is 15 inches. Table 1 gives the flow parameters and Fig. 1 shows the schematic diagram of the computational set up.

\section{Table 1 Flow parameters}

Free stream Mach number: $M_{\alpha}=3.5$

Free stream Reynolds numbers: $R e_{\alpha}=0.25,0.50^{*} 10^{6} /$ in.

Free stream temperature: $T_{\propto}=156.42^{\circ} \mathrm{R}$

Wall temperature: $T=476.0{ }^{\circ} \mathrm{R}$

Prandtl number: $P r=0.72$

Ratio of specific heats: $\gamma=1.4$

Local Reynolds number: $\sqrt{\operatorname{Re}_{x}}=\sqrt{\frac{U_{e} x}{v}}$

The nose region of the cone is modeled as a circle of the form

$$
\left(x-r_{0}\right)^{2}+y^{2}=r_{0}^{2}
$$

Here $r_{0}$ is the radius of the tip bluntness. The circular nose is smoothly merged with the cone by a tenth order polynomial. Simulations are performed for a nose radius of $r_{0}=0.001$ inches.

The grid stretches in the $\eta$ direction close to the wall and is uniform outside the boundary layer. In the $\xi$ direction, the grid is symmetric about the tip and very fine near the nose and is uniform in the flat region. The grid is uniform in the azimuthal direction. The outer boundary outside of the shock follows a parabola to capture the boundary layer accurately. Calculations were performed using a grid size of $(3001 * 251 * 361)$. Due to the very fine grid distributions in the azimuthal and axial directions near the nose, the allowable CFL number is limited to very small values and it becomes very expensive to compute the entire domain at once. To overcome this, calculations are performed in several steps. First, the computations are done near the nose region with a small CFL number of 0.01. Second, the flow properties in the middle of this domain are fed as inflow conditions for the next domain and the computations are carried out with a larger CFL number of 0.50 .

\section{A. Roughness}

A three dimensional periodic roughness element is placed on the surface of the cone close to the nose region. The shape of the roughness is in the form

$$
y_{c}(x, \theta)=h e^{-\sigma\left(\frac{x-x_{r}}{l}\right)^{2}} \cos m \theta
$$

Here $y_{c}$ is the height of the roughness normal to the surface of the cone, $h$ is the maximum height, $x_{r}$ is the axial location of the roughness, $m$ is the azimuthal wavenumber, $l$ is the length scale and $\sigma$ is a constant 
that determines the width of the roughness in the axial direction. The Fourier transform of the roughness shape is given by

$$
\begin{aligned}
\tilde{y}_{c}\left(k_{x}\right) & =\frac{1}{2 \pi} \int_{-\infty}^{\infty} y_{c}(x) e^{-i k_{x} x} d x \\
& =\bar{h} \frac{1}{2 \sqrt{\pi \sigma}} e^{-\frac{k_{x}^{2}}{4 \sigma}}
\end{aligned}
$$

Here the variables $k_{x}$ and $h$ are non-dimensionalised by the length scale $l$. Computations are performed for $m=40$ and for the values $x_{r}, h$, and $\sigma$ that are given in Table 2 .

Table 2 Location and the height of the roughness

\begin{tabular}{|c|c|c|c|}
\hline $\mathbf{x}_{\mathbf{r}}$ (in.) & $\mathbf{h}$ (in.) & $\boldsymbol{\sigma}$ & $\mathbf{h} / \boldsymbol{\delta}$ \\
\hline $\mathbf{0 . 5 0}$ & $\mathbf{0 . 0 0 1}$ & $\mathbf{0 . 0 1}$ & $\mathbf{1} \mathbf{6}$ \\
\hline
\end{tabular}

\section{B. Mean flow without roughness}

The mean flow density contours and the boundary layer profiles computed using the WENO code without the roughness elements are given in Figs. 2-7. The results are depicted for the unit Reynolds number of $0.25^{*} 10^{6}$ /inch. Figure 2(a) shows the density contours in the larger domain while Fig. 2(b) shows the flow field near the nose region. We see the strong shock along the windward plane and a weaker shock along the leeward plane. The shock angles are 21.0 and 30.0 degrees along the windward and the leeward planes, respectively. The shock is detached about 0.0001 inches from the nose of radius 0.001 inches. The maximum density near the nose occurs about 1 degree towards the windward plane from the nose tip for this angle of attack of 4.2 degrees. Figure 3 shows the density contours in the cross sectional plane at different axial locations $x=0.055$ and 7.55 inches. The figure clearly shows the accumulation and thickening of the boundary layer near the leeward plane. Very close to the tip, the boundary layer thickness increases gradually up to the leeward side. Further downstream, bulges start to form in a narrow region near the leeward plane. This is due to the accumulation of low speed fluid that is advected towards the leeward plane by the crossflow.

Figures 4-6 show the boundary layer density profiles at three azimuthal planes, windward $\theta=0^{\circ}, \theta=$ $90^{\circ}$, and leeward $\theta=180^{\circ}$. The profiles are displayed for the axial stations $x=0.50,1.0,2.0,4.0,6.0,8.0$, 10.0 and 14.0 inches from the nose tip. Figures 4-6 (a) depict the profiles including the shock in the physical coordinate (in inches), while Figs. 4-6 (b) show the boundary layer region. Figures 4-6 (c) show the boundary layer profiles in the similarity coordinate. We note that the vertical scales in these figures have been increased from Fig. 4 to Fig. 6 due to the increasing boundary layer thickness in the azimuthal direction. The boundary layer thicknesses at $x=6$ in. at the windward $\theta=0^{\circ}, \theta=90^{\circ}$, and leeward $\theta=180^{\circ}$ locations are $0.020,0.025$ and $0.12 \mathrm{in}$. respectively. As we discussed above, the boundary layer along the leeward plane is about 6 times thicker than that along the windward plane. We also observe that along the leeward planes the density profiles deviate from being smooth and develop bends in their shapes. This may influence the stability properties along the leeward plane.

It is known that the inviscid flow over a sharp cone at an angle of attack is conical in the azimuthal direction. The flow properties remain constant along a ray emanating from the apex. This allows seeking similarity solutions along the rays. Hence, the boundary layer equations for flow over a sharp cone at an angle of attack can be transformed into simplified partial differential equations that can be solved by marching in the azimuthal direction. The similarity equations are given in Ref. 10. It is interesting to see from Figs. 4(c) and 5(c) that the velocity profiles in similarity coordinates exhibit the expected similarity behavior. The boundary layer thickness in the similarity variable are about 5.0 and 6.0 along $\theta=0^{\circ}, \theta=$ 
$90^{\circ}$ planes. As we approach the leeward plane the parabolic assumption in the azimuthal direction breaks down and we loose the similarity behavior as is evident in Fig. 6(c). Figures 7(a) and 7(b) show the boundary layer velocity profiles along the inviscid streamlines and along the crossflow directions at an axial station of $x=8.0$ inches. The profiles are plotted for different azimuthal angles from $\theta=0$ to $180^{\circ}$. The boundary layer velocity profiles vary slowly up to $170^{\circ}$ and then increase steeply in the last $5^{\circ}$ near the leeward plane. It is also noticed that the boundary layer profiles become inflectional along the leeward plane. Figure 7(b) shows that the crossflow velocity increases slowly from the windward plane with increasing azimuthal angle, reaches the highest magnitude of 0.08 around 90 degrees and decreases slowly towards the leeward plane. It is also observed that substantial crossflow exists even at $\theta=175^{\circ}$ from the windward plane. Figures 8(a) and (b) display the contours of the computed crossflow Reynolds numbers for the unit Reynolds numbers of 0.25 and $0.50 * 10^{6} /$ inch. The maximum crossflow Reynolds numbers are about 800 at the end of the cone for the lower unit Reynolds number case and is about 1150 for the higher Reynolds number case. The crossflow Reynolds number reaches a value of about 400 close to $x=4.0$ and 2.0 inches respectively for the lower and higher Reynolds number cases. Experimental results of King(1992) predicted that transition on a cone at an angle of attack occurred at crossflow Reynolds numbers in the range of $400 \sim 700$.

\section{Linear stability and the transition onset}

Figure 9 shows the linear stability results obtained from the local parallel theory at the axial stations $x=2.0,5.0$ and 10.0 inches along the azimuthal direction, $\theta$. The figure gives the growth rates for two nondimensional frequencies $F$ over a range of azimuthal wavenumbers $m$. As expected the growth rates reach a maximum in the middle region due to the presence of strong crossflow in this region. The most amplified frequencies are about $50-60 \mathrm{kHz}$ at this unit Reynolds number of $0.25 * 10^{6} / \mathrm{inch}$. The azimuthal wavenumbers of the most unstable waves are gradually increasing from about $m=30$ at $x=2$ inches, to $m$ $=40$ at $x=5$ inches, and to $m=50$ at $x=10$ inches. This implies that as a traveling wave with a constant frequency propagates downstream, its wavelength in the azimuthal direction will gradually increase. The figures show that the growth rates for the traveling disturbances are about two times larger than that for the stationary disturbances The azimuthal wavenumbers of the most unstable stationary crossflow vortices are in the range of $m \sim 30-75$. The azimuthal wavenumbers of the most unstable waves range from 30 to 40 at $x=2$ inches, from 50 to 70 at $x=5$ inches, and from 60 to 70 at $x=10$ inches. Hence, the important question is, how will the wavelength of the stationary crossflow vortices change as they evolve downstream in three-dimensional flows? The results from the direct numerical simulation with roughness elements presented in the next section may yield some answer to this question. We also included the growth rate curves for a higher frequency $F=2.073 * 10^{-4}(85 \mathrm{kHz})$ in the Figs. 9(a) and (b). This frequency gives the earliest transition onset along the leeward plane. Figure 9(a) clearly shows that the growth rate is at a maximum near the leeward line at this location $x=2$ inches. It is also observed that the growth rate along the leeward plane is still comparable to the maximum growthrate in the crossflow region at $x=5$ inches.

Figure 10(a) shows the N-Factor traces obtained from the emalik $^{3 d}{ }^{16}$ code using the envelop method for the traveling disturbances for the case with a unit Reynolds number of $0.25 * 10^{6} /$ inch. Each trace is obtained by starting the computations near the neutral point for a fixed frequency and marching downstream along the group velocity direction while seeking maximum growth rate at each station. Each trace ends at the location where $N=11$. N-Factor computations performed by marching along the inviscid streamlines yield almost the same traces and the $\mathrm{N}$-Factor values. The figure shows the results for the frequencies that reached the largest N-Factor at that location. The unstable frequency for this case is about $50 \mathrm{kHz}$. The transition front takes an arc shape with the transition front far forward in the crossflow region and further backward near the windward and leeward regions. The earliest detection of transition occurred along the $120^{\circ}$ ray at the location $x=6$ inches. The predicted transition near the leeward side is about $x=$ 10 inches and the transition along the $20^{\circ}$ ray is at $x=15$ inches. Comparison with Fig. 8(a) shows that the early transition happens in the region where crossflow Reynolds number is high. Figure 10(b) 
similarly depicts the traces for the stationary disturbances. The N-Factors are smaller than 11 for this case within the computational domain except within the rays $110^{\circ}$ and $170^{\circ}$. The N-Factors at the end of the traces are also written in the figure. We also included the N-Factor trace (dark black line) obtained along the leeward plane. The unstable frequency for this trace is about $85 \mathrm{kHz}$ and the predicted transition onset occurs at $x=7$ inches. As we discussed previously, the transition occurs earlier along the leeward line than in the region close to the leeward plane. In the experiments ${ }^{12}$, contrary to the prediction based on the $\mathrm{N}$-Factor method, the transition front gradually moved upstream from the windward side to the leeward side. One possible scenario may be that the turbulent wedge that forms on the leeward line spreads and contaminates the region close to it.

The maximum N-Factor for the stationary disturbances obtained near the $40^{\circ}$ ray is about 3 . The early detection of transition occurred along the $135^{\circ}$ ray at the location $x=13$ inches. Additional important information we need to gather from Figs. 10 (a) and (b) are about the start and end points of each NFactor trace. The starting point indicates the neutral point for that frequency. The neutral points for stationary disturbances vary along the line $x=1.0, \theta=60^{\circ}$ to $x=6.5 \mathrm{in}, \theta=20^{\circ}$. The corresponding points for the traveling disturbances are along the line $x=0.5, \theta=60^{\circ}$ to $x=2.5$ in, $\theta=20^{\circ}$. The N-Factor traces which follow the group velocity direction curve upwards towards the leeward plane. For example, the traces that start near $x=1.0$ inch end closer to the leeward plane. The trace that starts at $x=4.5$ inches ends at $x=15$ inches and $\theta=90^{\circ}$. If this analysis is correct, it implies that if we place the roughness elements upstream of any one of the traces, say at a fixed $x$ location, stationary crossflow vortices will not develop efficiently downstream of this trace. We will compare this conjecture with the simulation results in the next section. Figure 11 shows the transition onset fronts caused by the traveling and the stationary disturbances for different unit Reynolds numbers of $0.25 * 10^{6} / \mathrm{inch}$ and, $0.50 * 10^{6} /$ inch. As expected, transition onset due to traveling disturbances occurs upstream of that due to stationary disturbances. The earliest detection of transition occurred around $x=3$ and $x=7$ inches due to traveling and stationary disturbances respectively for the unit Reynolds number of $0.50 * 10^{6} /$ inch case and occurred around $x=6.5$ and 13.0 inches for the lower unit Reynolds number of $0.25^{*} 10^{6} /$ inch case. However, these relative locations depend on amplification rates and in practice are dependent on the free stream disturbance levels and the roughness distribution on the surface.

\section{The generation and the evolution of stationary crossflow vortices.}

The generation and the evolution of stationary crossflow vortices in a supersonic boundary layer at an angle of attack were simulated by placing isolated roughness elements on the surface of the cone, Fig. 1. The parameters of the roughness elements are given in Table 2. The roughness is placed at $x_{r}=0.50$ in., the azimuthal wavenumber is $m=40$ and the height of the roughness is $h=0.001$ inches. Figure 12 shows the contours of the axial velocity, $u$, in the $(x, r \theta)$ plane. The figure shows the $u$-velocity contour in the plane at a constant normal grid number of 40 . This plane approximately goes through the middle of the boundary layer. The image can be considered as the footprints of the stationary crossflow vortices that originated from the roughness elements. The figure clearly displays that the crossflow vortices that are originating from the roughness elements as they evolve towards the leeward plane. It was surprising to observe that no footprints are observed below the $120^{\circ}$ ray. At the last station, $x=8$ inches, the vortices are confined between the ray $135^{\circ}$ and the leeward plane. Figures 13 and 14 show the contours of the axial velocity, $u$, at different cross sections $x=3.2,4.8,6.3$ and 7.9 inches. Figure 13 displays the contours around the cone surface in the Cartesian coordinates while Fig. 14 depicts the results in the azimuthal angle, $90^{\circ}<\theta<180^{\circ}$, and the normal distance to the cone surface. In Fig. 14, the windward side is towards the right side of the plot and the leeward side is on the left side. As we discussed previously, it is seen that the vortices are confined to regions close to the leeward plane. The figures clearly display the co-rotating crossflow vortices near the leeward side. The vortices are rotating towards the windward side. At $x=3.2$ inches, vortices are observed between 120 and 155 degrees. As the vortices evolve downstream, they grow in magnitude and shift more towards the leeward plane. At $x=6.3$ inches, the vortices reside between 130 and 165 degrees. It is also observed from Figs. 14(c) and (d) that the 
vortices grow in amplitude as they propagate from $x=6.3$ to $x=7.8$ inches, but they do not shift to the left and remain at the same azimuthal locations.

Figure 15 shows the amplitude of the fluctuations of the streamwise velocity, $u$, very near the roughness elements at stations $x=0.60,0.75,1.0$ and 1.7 inches (The velocities are non-dimensionalised by $\sqrt{R T_{\infty}}$ ). The windward and the leeward planes are on the left and right sides of the plots, respectively. The $u$-velocity fluctuations are plotted at different grid locations, 10,30,50 and 60 . The corresponding normal heights for these grid locations in inches are: $(0.001,0.0033,0.0056,0.0068),(0.0011,0.0037$, $0.006,0.0075),(0.0013,0.0042,0.007,0.0085)$ and $(0.0016,0.0053,0.0089,0.011)$ at $x=0.60,0.75,1.0$ and 1.7 inches, respectively. Figure 15(a) shows that disturbances are generated across the entire region from the windward to leeward planes with the same wavelength, $9^{\circ}$, as the roughness elements. The maximum fluctuations occur very close to the surface and between 20 to 80 degrees. The amplitude of the maximum perturbations nondimensionalised by the freestream velocity is about 0.017 . Figures $15(\mathrm{~b})$, (c) and (d) show that the amplitudes first decrease with increasing $x$ up to $x=1.0$ inches, and increase beyond $x=1.0$ inches. This agrees with the linear stability theory that the neutral points are located near $x=1.0$ inches. The amplitude of the perturbations decreases to 0.005 at $x=1.0$ inches. If we use this as the estimate for the initial amplitude of the unstable stationary crossflow vortices, we obtain the receptivity coefficients as

$$
\left(\frac{u}{U_{\infty}}\right)_{\text {neutral }}=0.005
$$

for the roughness height of 0.001 inches. The figures also show that within a short distance from the roughness the vortices have moved towards the leeward side with increasing $x$. The maximum perturbations occur close to 120 degrees at $x=1.7$ inches.

Figure 16 displays the axial $\mathrm{u}$-velocity at five downstream axial locations and at different normal heights in inches: $(x=1.7, y=0.011),(x=3.2, y=0.014),(x=4.8, y=0.019),(x=6.3, y=0.024)$ and $(x$ $=7.9, y=0.025)$. The different heights correspond to the locations where the maximum perturbations are detected. Figure 16(a) and Fig. 15(d) are displayed for the same axial station $x=1.7$ inches. The curves show that beyond $x=1.0$ inches the perturbations continue to increase and the vortices move towards the leeward plane. It is also observed that no perturbations exist below 130 degrees beyond $x=6.3$ inches (note that vortices do not shift appreciably between $x=6.3$ and 7.9 inches). At this point we have not related the growthrate, wavelength and the azimuthal wavenumber obtained from the linear locally parallel stability calculations to the perturbation field obtained from the simulation. However, if we estimate the wavelength of the perturbations near the maximum oscillations by measuring the distance between two peaks, we obtain the wavelengths as $10.2,10.0,7.0,6.4$ and 6.4 degrees at $x=1.7,3.24 .8$, 6.3 and 7.9 inches, respectively. The corresponding wavenumbers are $m=35,36,51,56$, and 56. The figures clearly show that the wavelengths are not uniform in the azimuthal direction. Hence these numbers have to be taken as the average wavelength of the perturbations in the azimuthal direction near the leeward side. Figure 9 shows that the azimuthal wavenumbers of the most amplified waves are about 30,50 , and 60 near $\theta=150^{\circ}$ at $x=2,5$, and 10 inches respectively. It is seen that the average wavenumbers estimated from the simulation are in the same range as that predicted from the stability theory at these locations. The wavenumber of the roughness distribution is $m=40$. It is not clear yet how the boundary layer modifies the wavenumbers of the crossflow vortices from their initial value of 40 as they evolve downstream. This may be due to the fact that the stability characteristics of the boundary layer, the wavenumber and the growth rate, vary along the azimuthal direction. Hence, as the vortices evolve downstream their amplitudes and wavelengths are modified along the axial and the azimuthal directions. 


\section{Discussion and Conclusions}

We investigated the stability, transition onset, and the generation of crossflow vortices in a threedimensional supersonic boundary layer over a sharp tipped $7^{\circ}$ cone at an angle of attack of $4.2^{\circ}$. Mean flow computations showed that the boundary layer along the leeward plane is very inflectional and is about 6 times thicker than that along the windward plane. The boundary layer profiles are self-similar along each ray except near the leeward planes. Simulations also revealed that the crossflow peaks around the middle region between the windward and the leeward planes and it becomes weaker near the leeward and the windward sides. However, substantial crossflow velocity exists up to $175^{\circ}$ from the windward plane.

The linear stability results showed that the traveling disturbances are about twice as unstable as compared to the stationary disturbances. The azimuthal wavenumbers for the unstable traveling disturbances are in the range of 25-45 and they are in the range of 40-75 for the stationary disturbances. The N-Factor computations predicted that transition would occur further forward in the middle of the cone compared to that along the windward and the leeward planes. It was also observed that the boundary layers near the leeward plane are more unstable compared to the boundary layers near the windward plane. Transition occurs farther downstream due to the stationary disturbances compared to what would be caused by the traveling disturbances. However, these relative locations are dependent on the free stream disturbance levels and the roughness distribution on the surface. The N-Factor traces indicated that the transition is caused by the disturbances originating from regions closer to the windward side of the cone.

The direct numerical simulation with the three-dimensional elements located near the cone tip revealed that crossflow vortices that are originating from these roughness elements evolve towards the leeward side of the cone. The simulation also showed that at a short distance downstream of the roughness, disturbances are generated between the windward and the leeward planes with the same wavelength as the roughness distribution. The maximum disturbances occur in the middle part of the cone. The perturbations first decay up to the neutral points and then increase to larger values further downstream. The vortices migrate towards the leeward plane with increasing axial distance. There exist no perturbations between the windward plane and the $120^{\circ}$ ray. These conclusions might change if we move the location of the roughness elements downstream. One important conclusion that could be drawn from this study is that if one wants to design a crossflow transition or a flow control experiment similar to Discrete Roughness Elements (DREs) ${ }^{3}$, it is prudent to place the roughness elements along a ray closer to the windward plane or along the neutral line predicted from the stability theory. We have not related the growth rates and the wavelengths obtained from the linear stability theory to the perturbations field obtained from the simulation. Some preliminary analysis revealed that the azimuthal wavenumbers on the average increase in the downstream direction. The average azimuthal wavenumbers of the crossflow vortices near the leeward plane estimated from the simulation are within the range of the most amplified stationary disturbances in this region. The intriguing question of how to decompose the computed flow field into normal modes type disturbances in a three-dimensional flow will be analyzed in the future.

\section{References}

1. Gregory, N., Stuart, J., and Walker, W., "On the Stability of Three-dimensional Boundary Layers with Application to the Flow Due to a Rotating Disc," Philosophical Transactions of the Royal Society of London, 1955, pp. 155-198.

2. Deyhle, H., and Bippes, H., "Disturbance Growth in an Unstable Three-Dimensional Boundary Layer and its Dependence on Environmental Conditions," Journal of Fluid Mechanics, Vol. 316, Jun. 1996, pp. 73113.

3. Saric, W., Carillo, R., and Reibert, M., "Leading Edge Roughness as a Transition Control Mechanism," AIAA Paper 98-0781, Jan. 1998. 
4. Malik, M., Li, F., and Chang, C., "Crossflow Disturbances in Three-Dimensional Boundary Layers; Nonlinear Development, Wave Interaction and Secondary Instability," Journal of Fluid Mechanics, Vol. 268, Jun. 1994, pp. 1-36.

5. Saric, W. S., Reed, H. L., and White, E. B., "Stability and Transition of Three-Dimensional Boundary Layers," Annual Review of Fluid Mechanics, Vol. 35, Jan. 2003, pp. 413-440.

6. Lees, L., and Lin, C. C., "Investigation of the Laminar Boundary Layer in a Compressible Fluid," NACA Technical Note, Vol. 83, Sept. 1946, pp. 115.

7. Lees, L., and Reshotko, E., "Stability of Compressible Boundary Layer," Journal of Fluid Mechanics, Vol. 12, 1962, pp. 455-477.

8. Mack, L., "Boundary Layer Stability Theory," JPL Report No. 900-277 Rev. A, Pasadena, Calif, Nov. 1969.

9. Balakumar, P., and Reed, H. L., "Stability of Three-Dimensional Supersonic Boundary Layers," Physics of Fluids, Vol. 3, No. 4, April 1991, pp. 617-632.

10. Malik, M. R., and Balakumar, P., "Instability and Transition in Three-Dimensional Supersonic Boundary Layers," AIAA Paper 1992-5049, 1992.

11. Chen, F. J., Malik, M. R., Beckwith, I. E., "Comparison of Boundary Layer transition on a Cone and Flat Plate at Mach 3.5," AIAA-88-0411, 1988

12. King, R., A., "Three-dimensional Boundary Layer Transition on a Cone at Mach 3.5", Experiments in Fluids, 13, 305, 305-314, 1992.

13. Cattafesta, L. N., Iyer, V., Masad, J. A., King, R. A., and Dagenhart, J. R., “Three-Dimensional Boundary Layer Transition on a Swept Wing at Mach 3.5," AIAA Journal 33, 1995.

14. Jeyasingham, S., and Balakumar, P., "Stability of Three-Dimensional Compressible Boundary Layers," AIAA Paper 1999-0406, 1999.

15. Jeyasingham, S., "Stability of Three-Dimensional Compressible Boundary Layers," M.S Thesis, Dept. of Aerospace Engineering, Old Dominion University, Norfolk, Virginia, 1999.

16. Malik, M. R., "eMalik3d: An $\mathrm{e}^{\mathrm{N}}$ Code for Three-Dimensional Flow over Finite-Swept Wings", High Technology Report No. HTC-9502, 1995.

17. P. Balakumar, "Receptivity of Supersonic Boundary Layers Due To Acoustic Disturbances Over Blunt Cones ," ALAA Paper 2007-4491, 2007.

18. Shu, Chi-Wang, "Essentially Non-Oscillatory and Weighted Essentially Non-Oscillatory Schemes for Hyperbolic Conservation Laws," NASA/CR-97-206253 and ICASE Report N0. 97-6

19. Atkins, H. L., "High-Order ENO Methods for the Unsteady Compressible Navier-Stokes Equations," AIAA Paper 91-1557, 1991.

20. Balakumar, P., Zhao, H., and Atkins, H., "Stability of Hypersonic Boundary_layers Over a Compression Corner," AIAA Paper 2002-2848, 2002. 


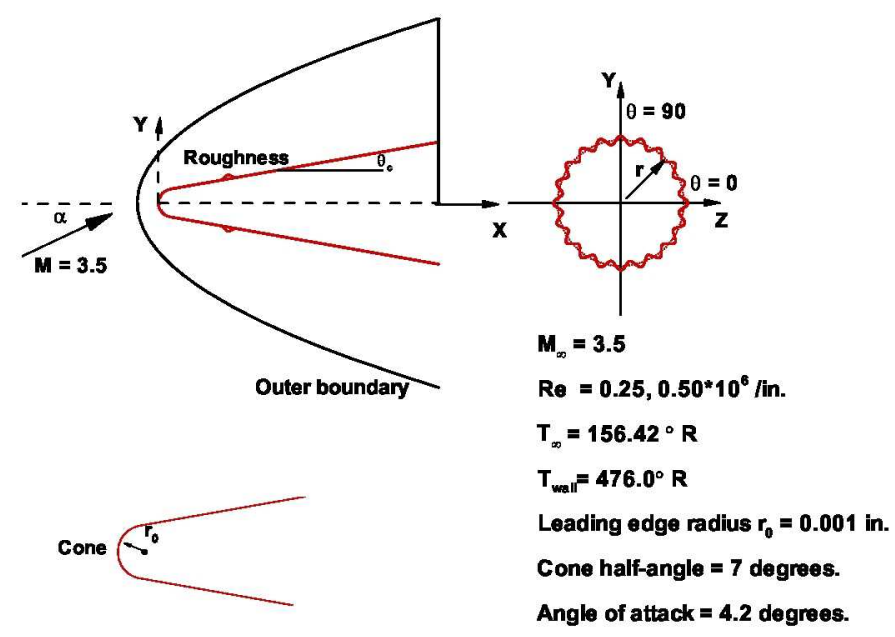

Figure 1. Schematic diagram of the computational model.

(a)

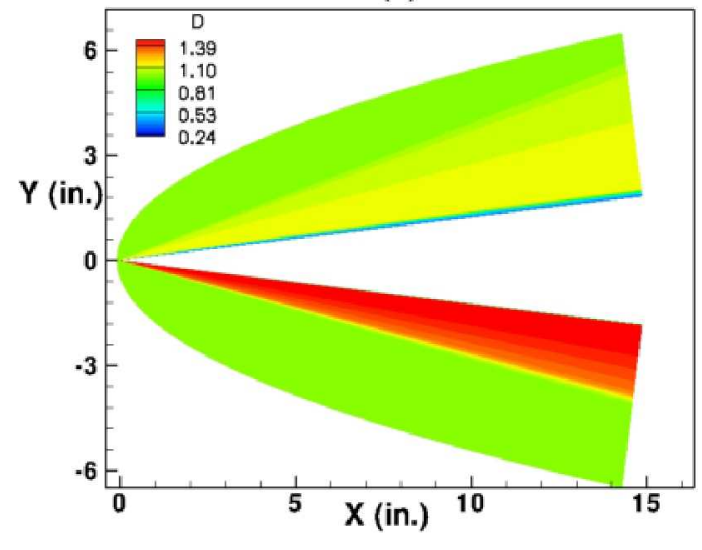

(b)

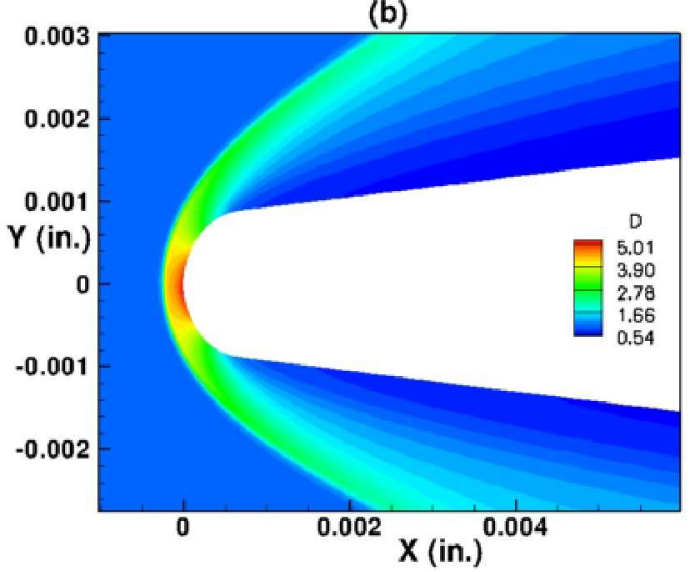

Figure 2. Contours of the density for flow over a 7-degree cone with a blunted leading edge at $M=3.5$ and at an angle of attack of 4.2 degrees, $R e=0.25 * 10^{6} / \mathrm{in}$.

(a) $x=0.055$ in.

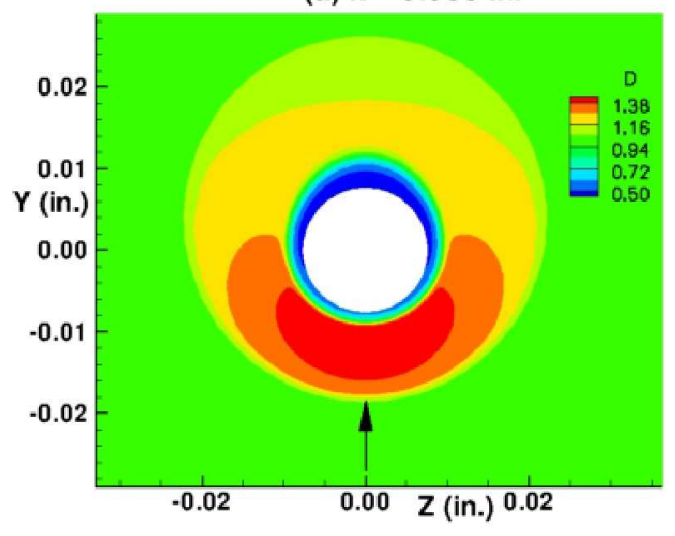

(b) $x=7.55$ in.

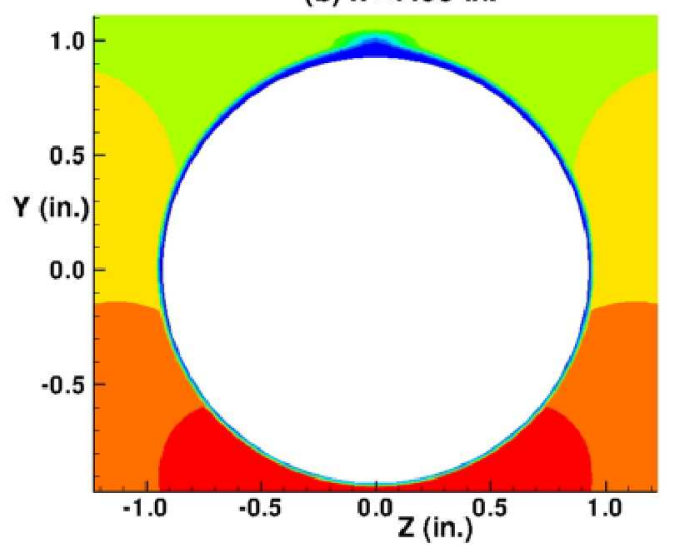

Figure 3. Contours of the density in the cross sectional planes at different axial locations.

11 of 16

American Institute of Aeronautics and Astronautics AIAA-2009-3555 
(a) windward side

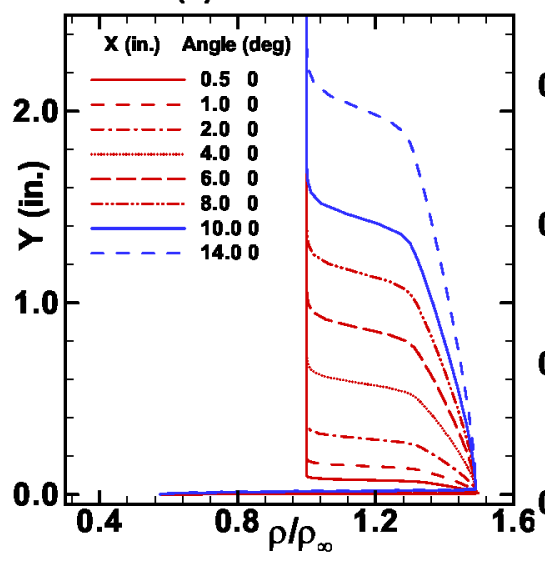

(b)

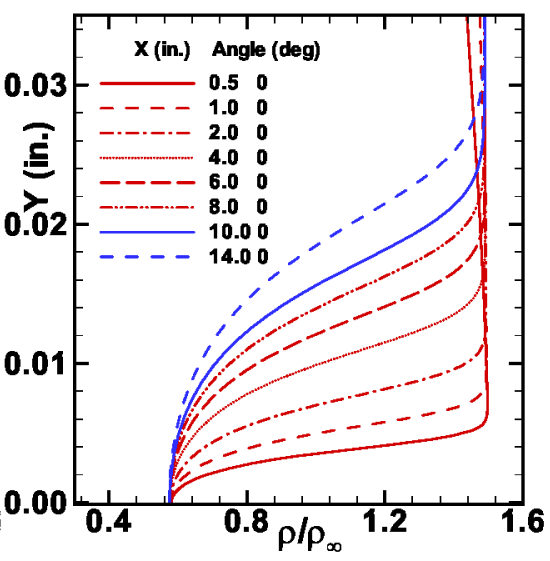

(c)

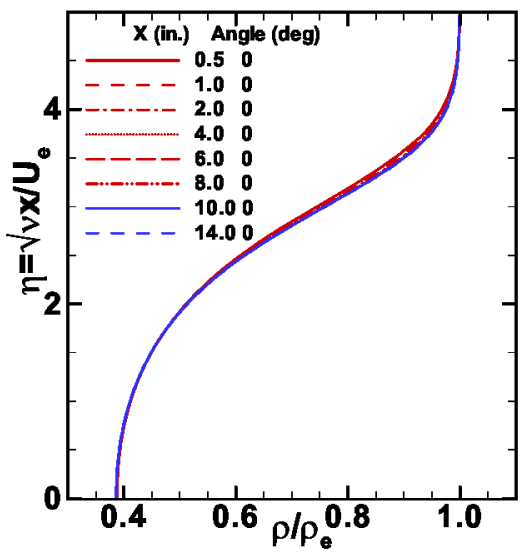

Figure 4. Density profiles along the windward side at different axial locations.

(a) azimuthal angle 90 deg

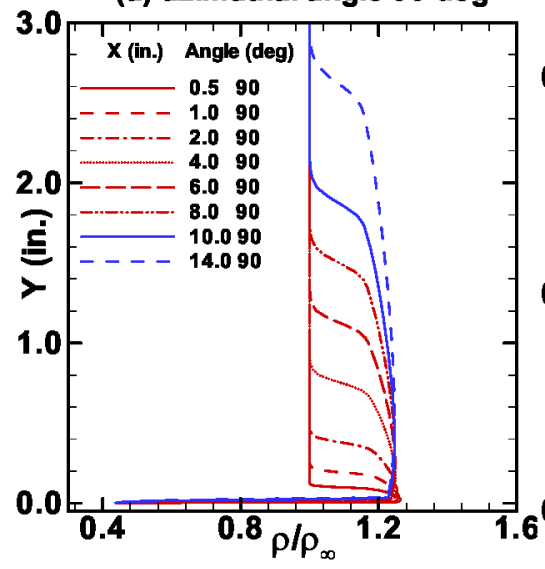

(b)

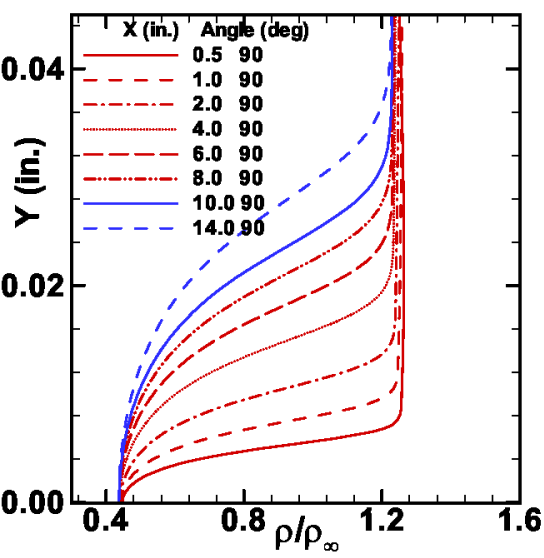

(c)

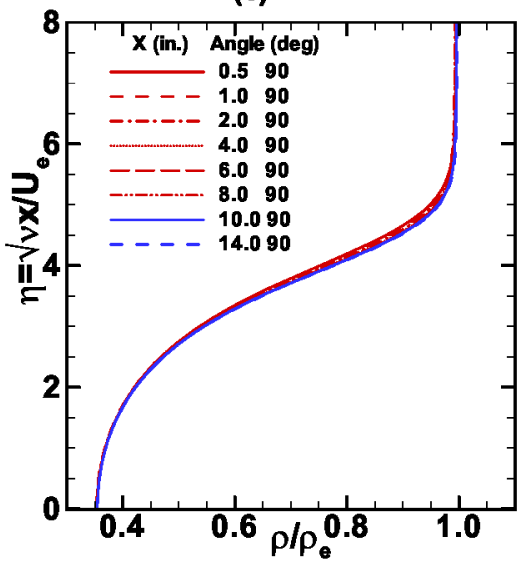

Figure 5. Density profiles along the azimuthal direction of 90 degrees at different axial locations.

(a) leeward side

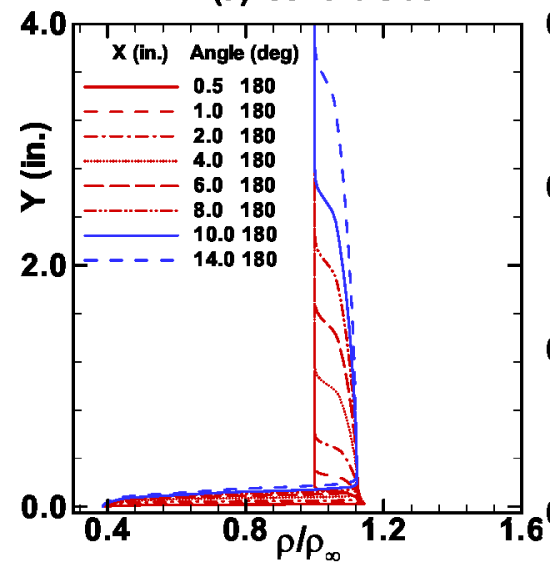

(b)

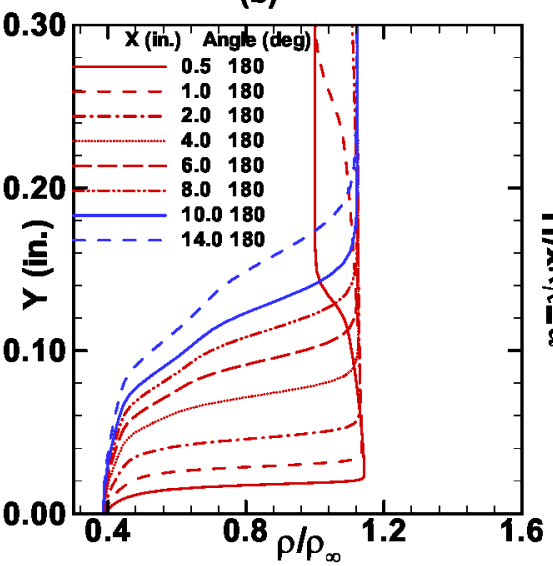

(c)

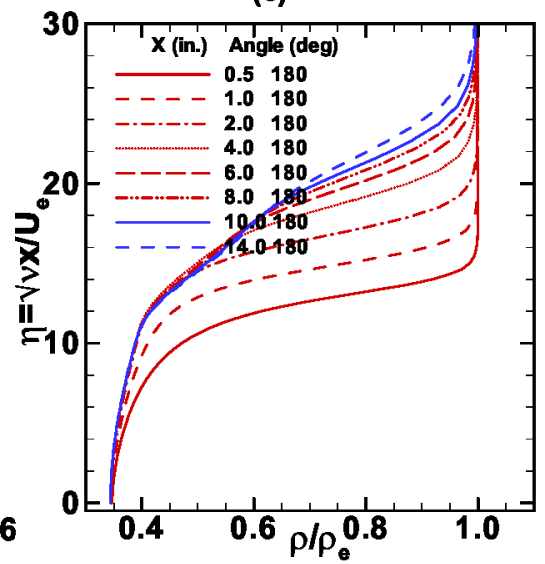

Figure 6. Density profiles along the leeward side at different axial locations.

12 of 16

American Institute of Aeronautics and Astronautics AIAA-2009-3555 
(a)

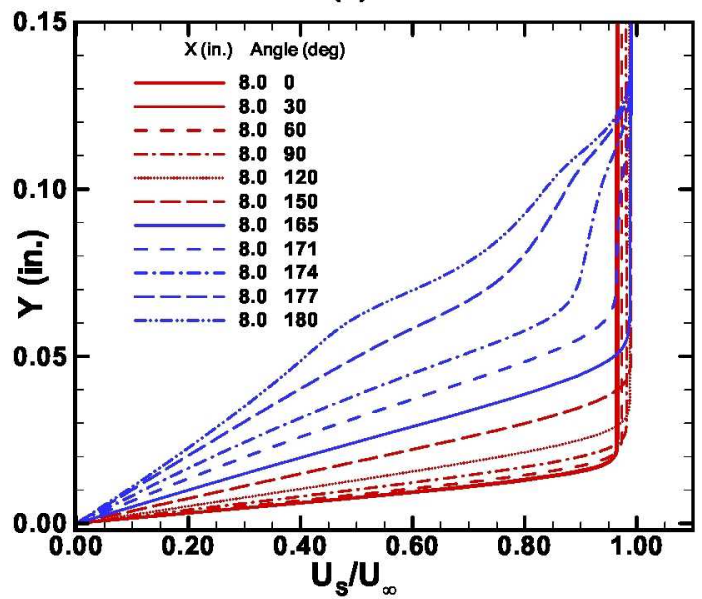

(b)

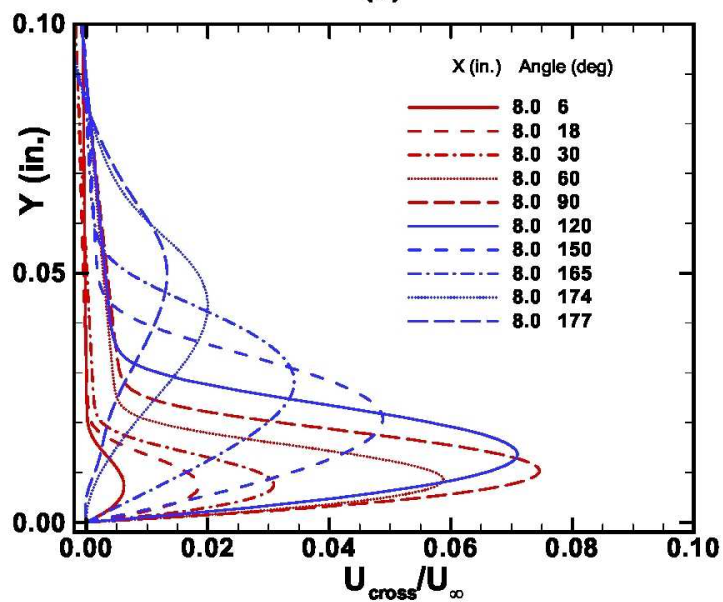

Figure 7. Boundary layer (a) streamwise velocity and (b) crossflow velocity profiles for different azimuthal angles at an axial location of $x=8$ inches.
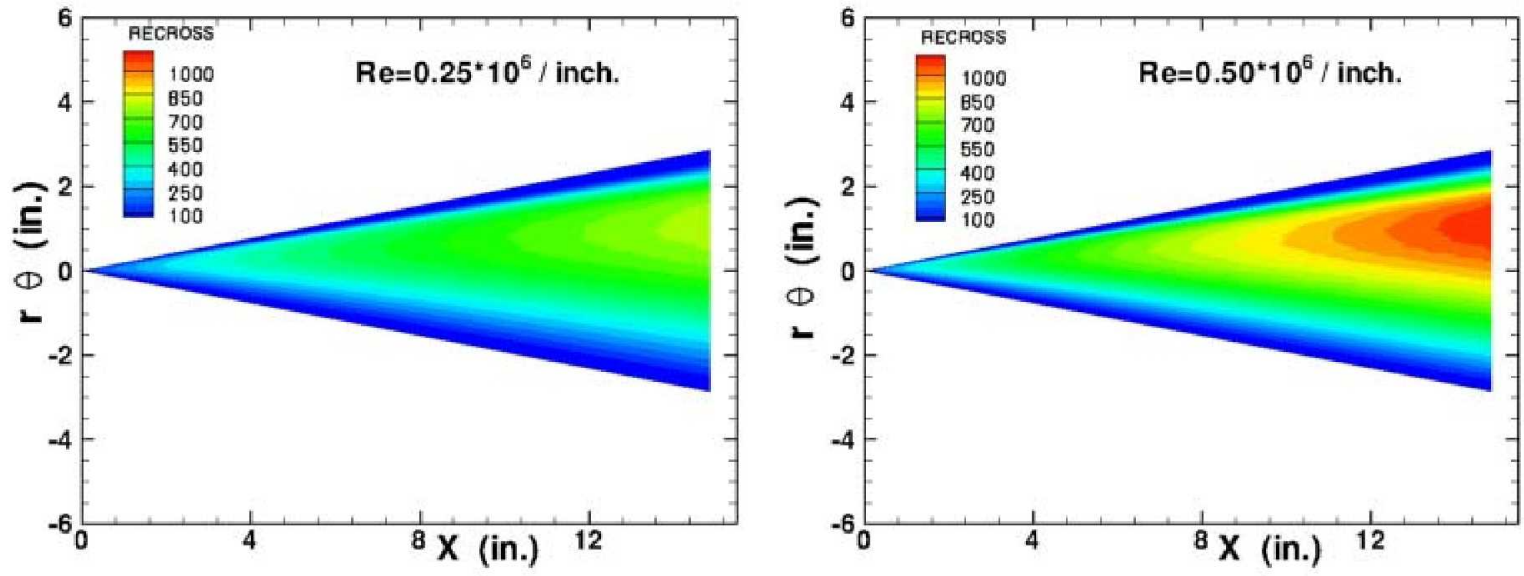

Figure 8. Crossflow Reynolds number. $M=3.5, R e=0.25$ and $0.50 * 10^{6} /$ inch, Angle of attack $=4.2$ degrees.

(a) $X=2$ in.

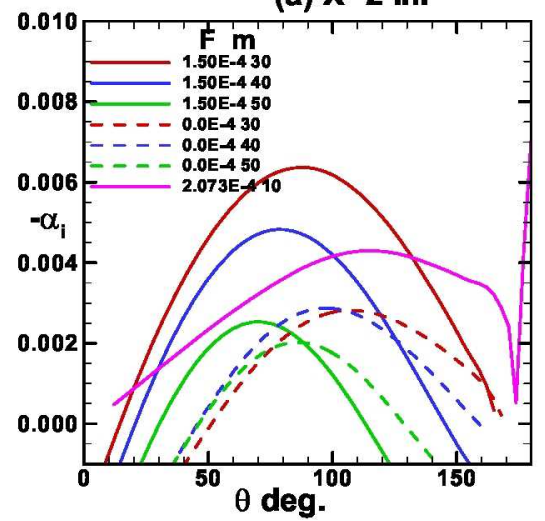

(b) $X=5$ in.

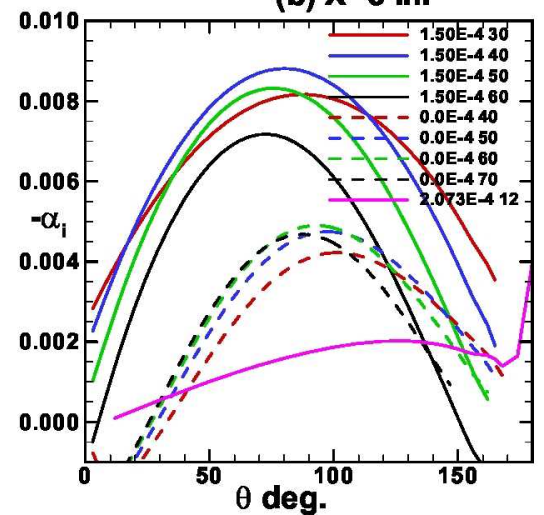

(c) $X=10$ in.

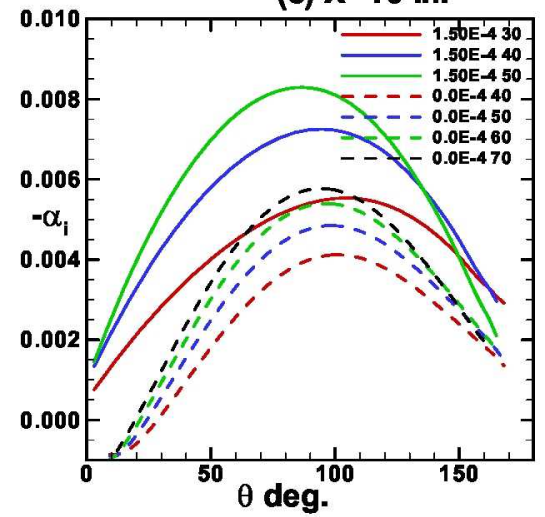

Figure 9. Growth rates for traveling and stationary disturbances at the axial locations $x=2,5$, and 10 inches. $\operatorname{Re}=0.25 * 10^{6} /$ inch.

13 of 16

American Institute of Aeronautics and Astronautics AIAA-2009-3555 
(a)

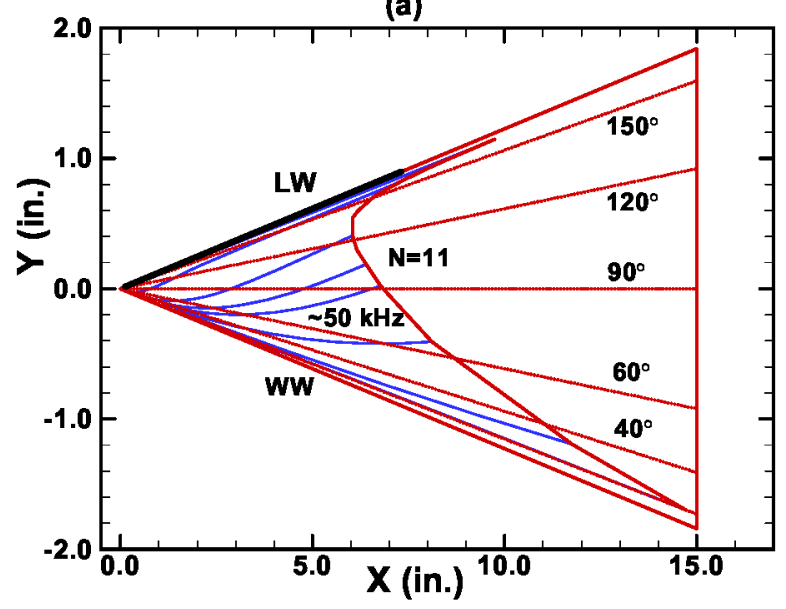

(b)

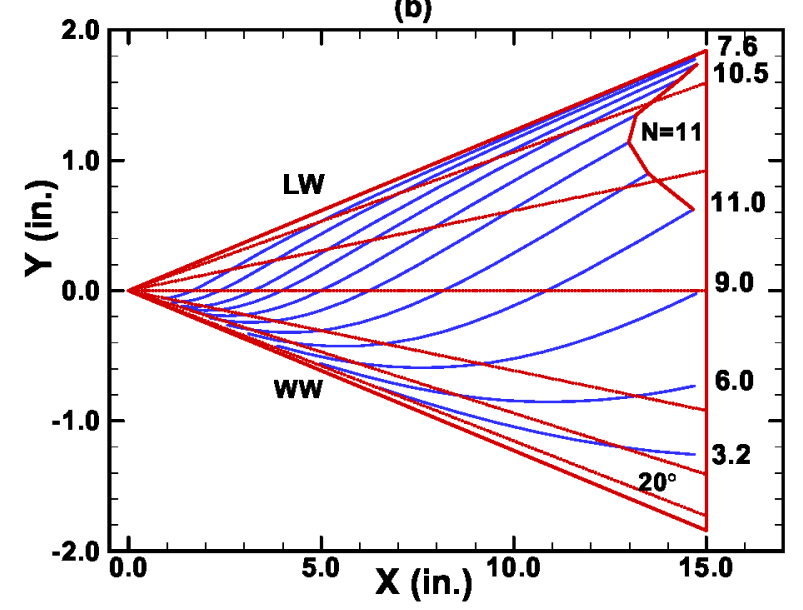

Figure 10. N-Factor traces for (a) traveling and (b) stationary disturbances. $R e=0.25^{*} 10^{6} / \mathrm{in}$.

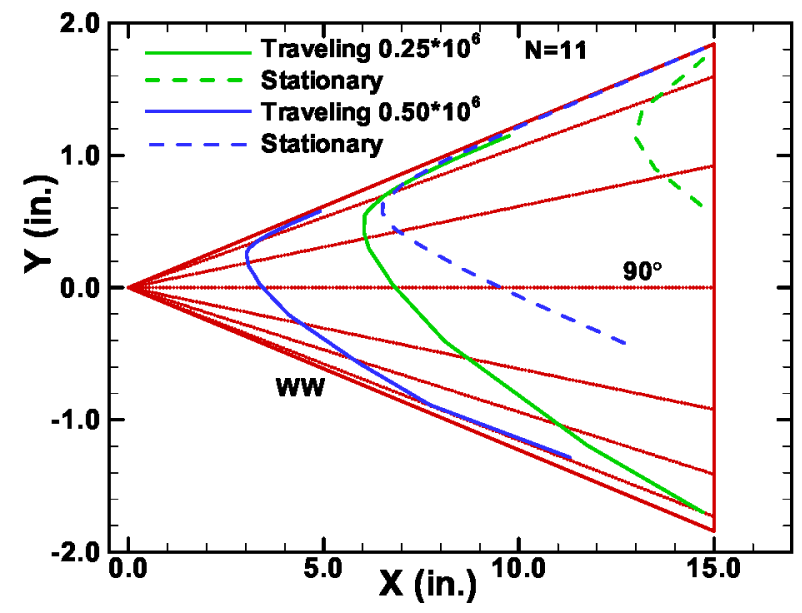

Figure 11. Transition onsets for traveling and stationary disturbances for the unit Reynolds numbers of $\operatorname{Re}=0.25$ and $0.50 * 10^{6} /$ in.

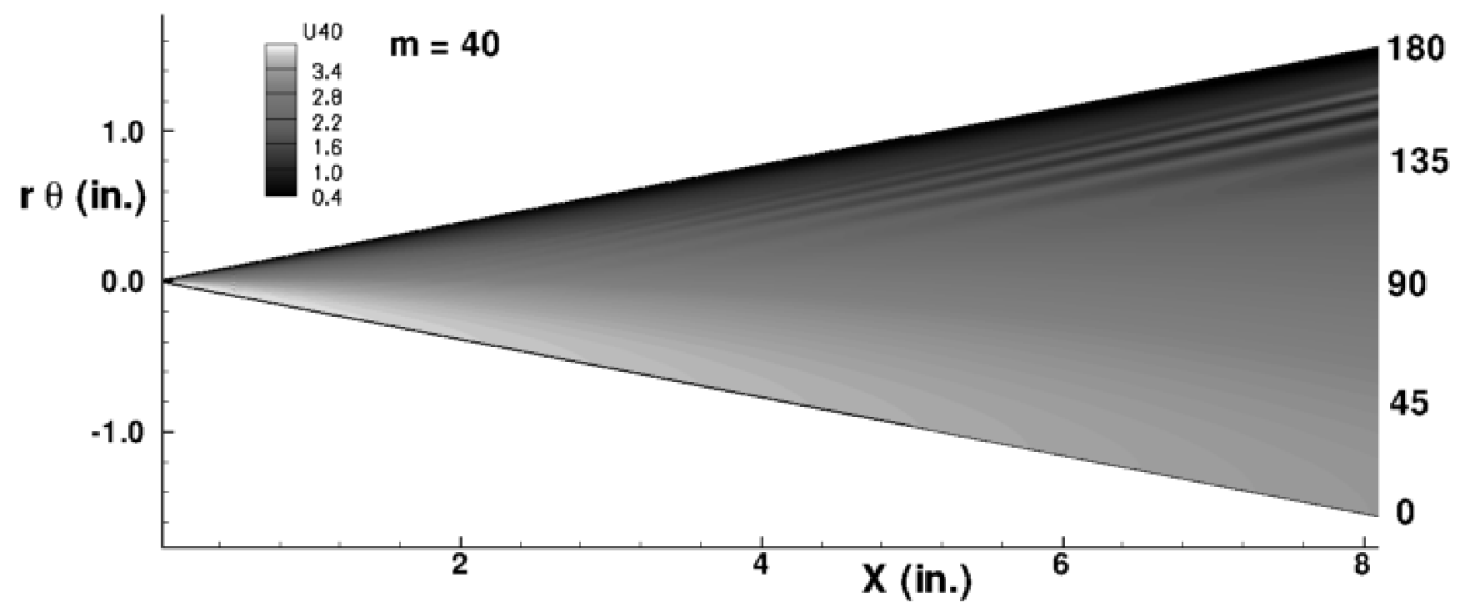

Figure 12. Contours of axial velocity, $u$, in the $x-\theta$ planes. $R e=0.25 * 10^{6} /$ inch, $m=40, h=0.001$ in., $x_{r}=0.5$ in. 
(a) $X=3.2$ in.

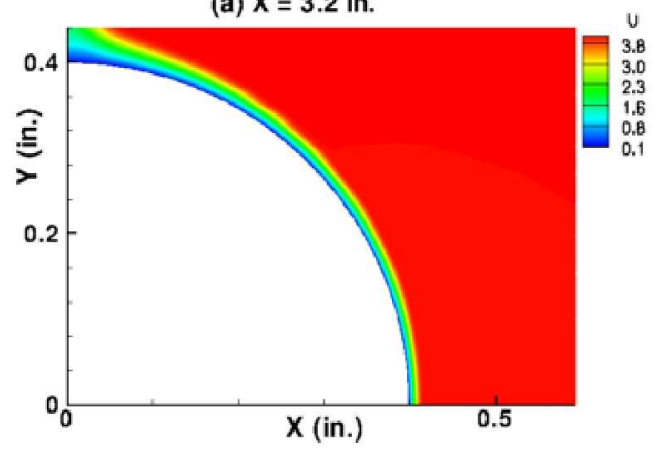

(c) $X=6.3$ in.

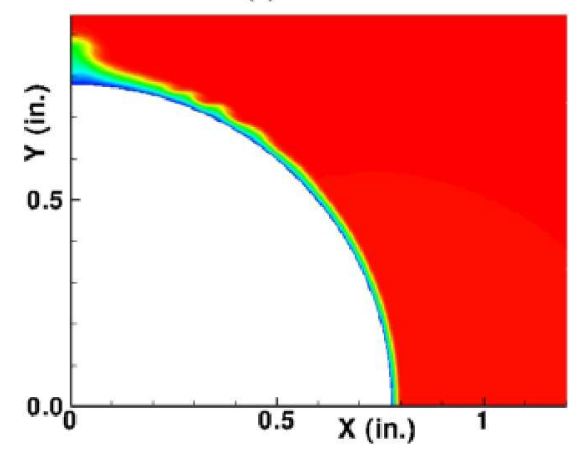

(b) $X=4.8$ in.

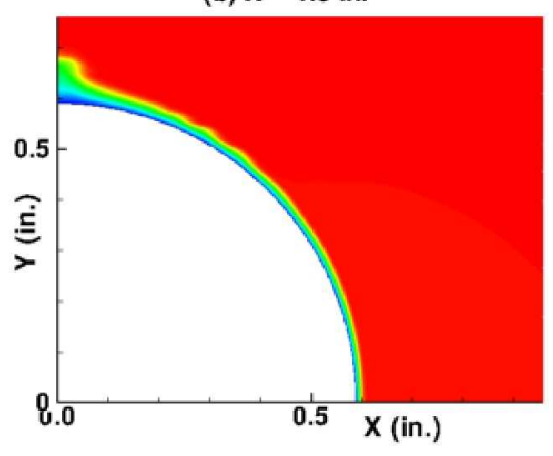

(d) $X=7.9$ in.

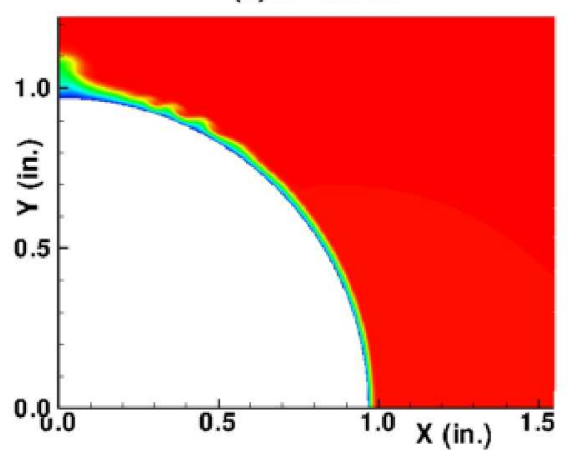

Figure 13. Contours of the axial velocity in the cross planes at different axial locations.

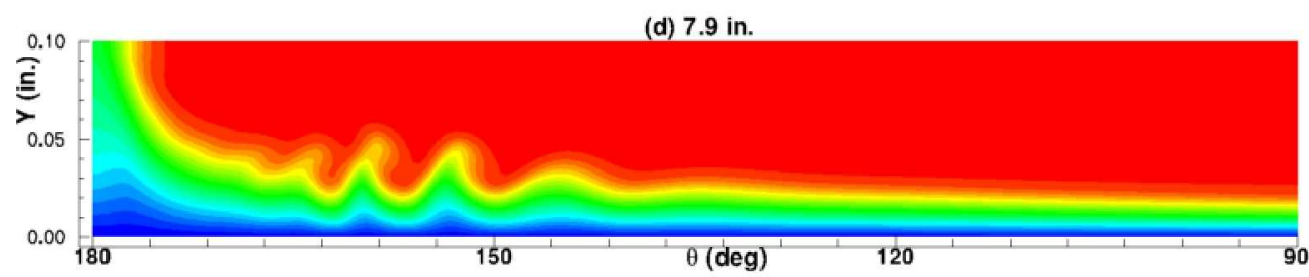

(c) 6.3 in.

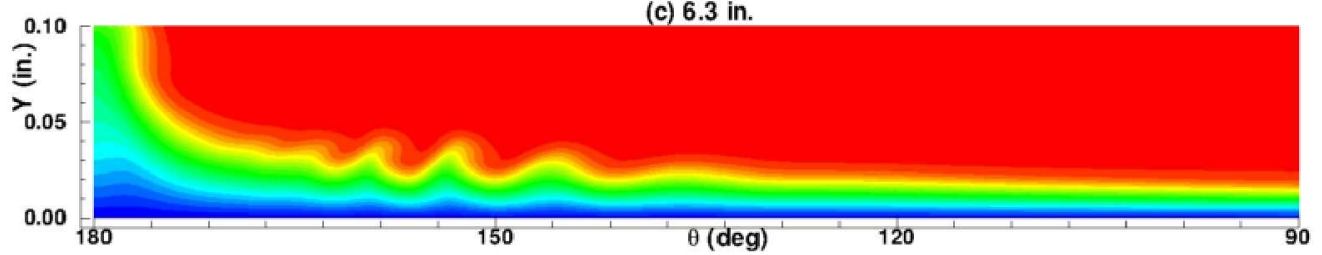

(b) 4.8 in.

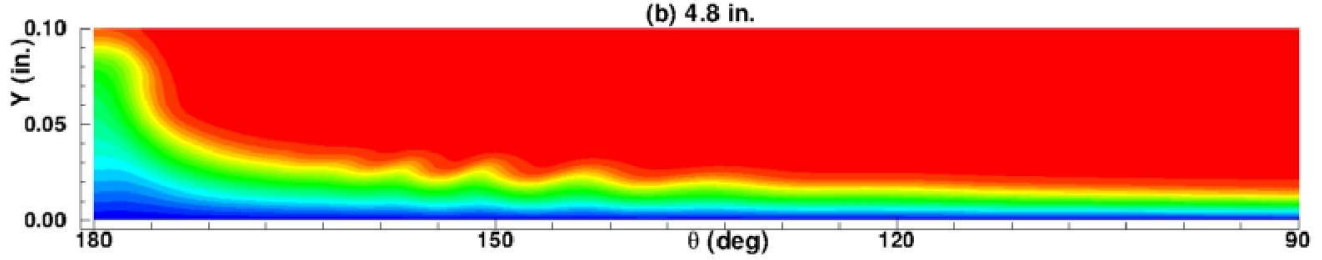

(a) 3.2 in.

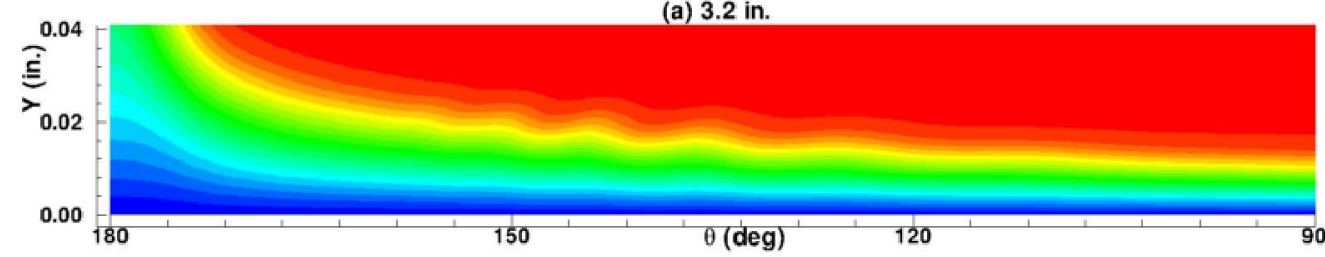

Figure 14. Contours of the axial velocity in the cross planes at different axial locations.

15 of 16

American Institute of Aeronautics and Astronautics AIAA-2009-3555 

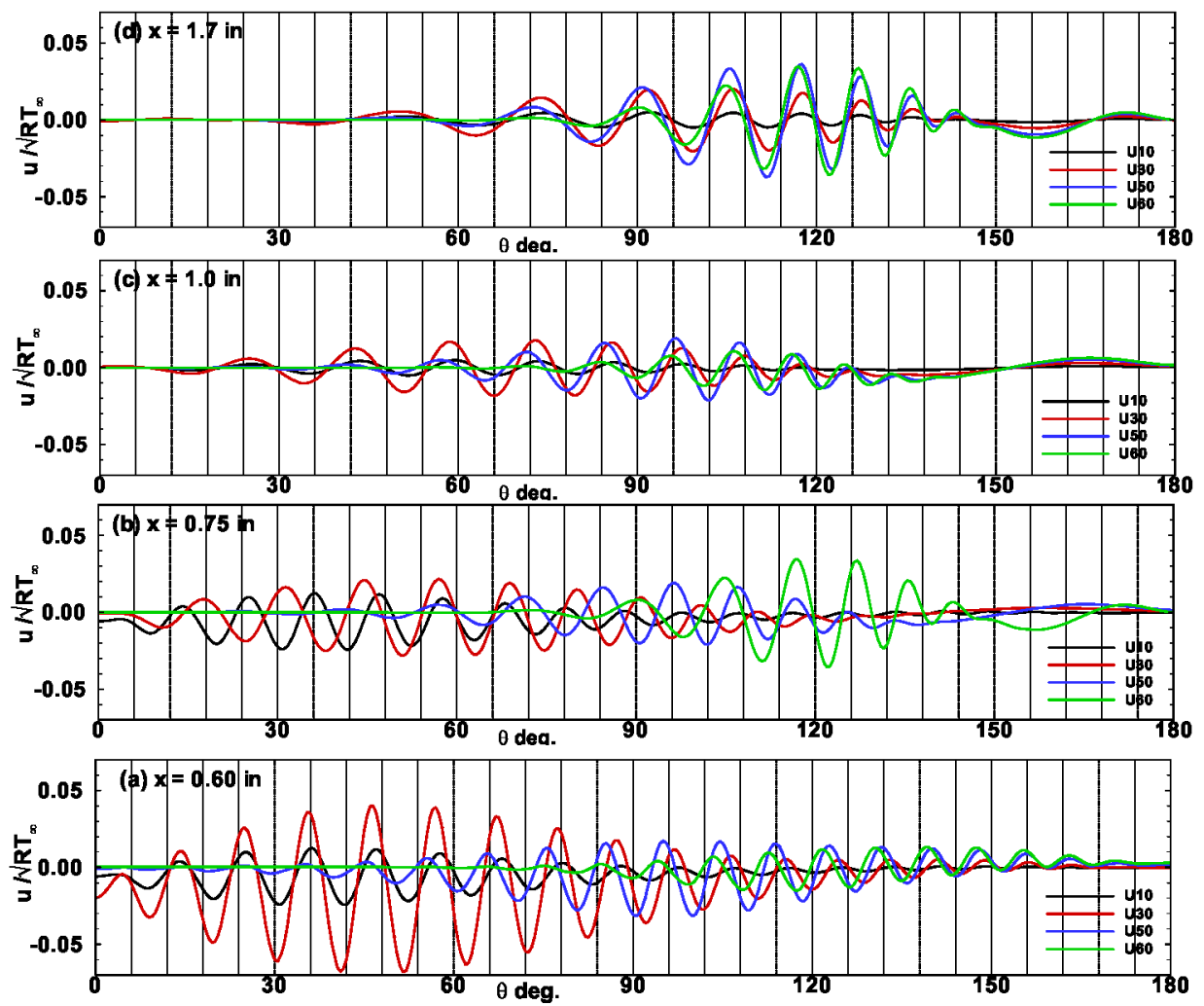

Figure 15. Contours of the axial velocity in the cross planes at different axial locations.
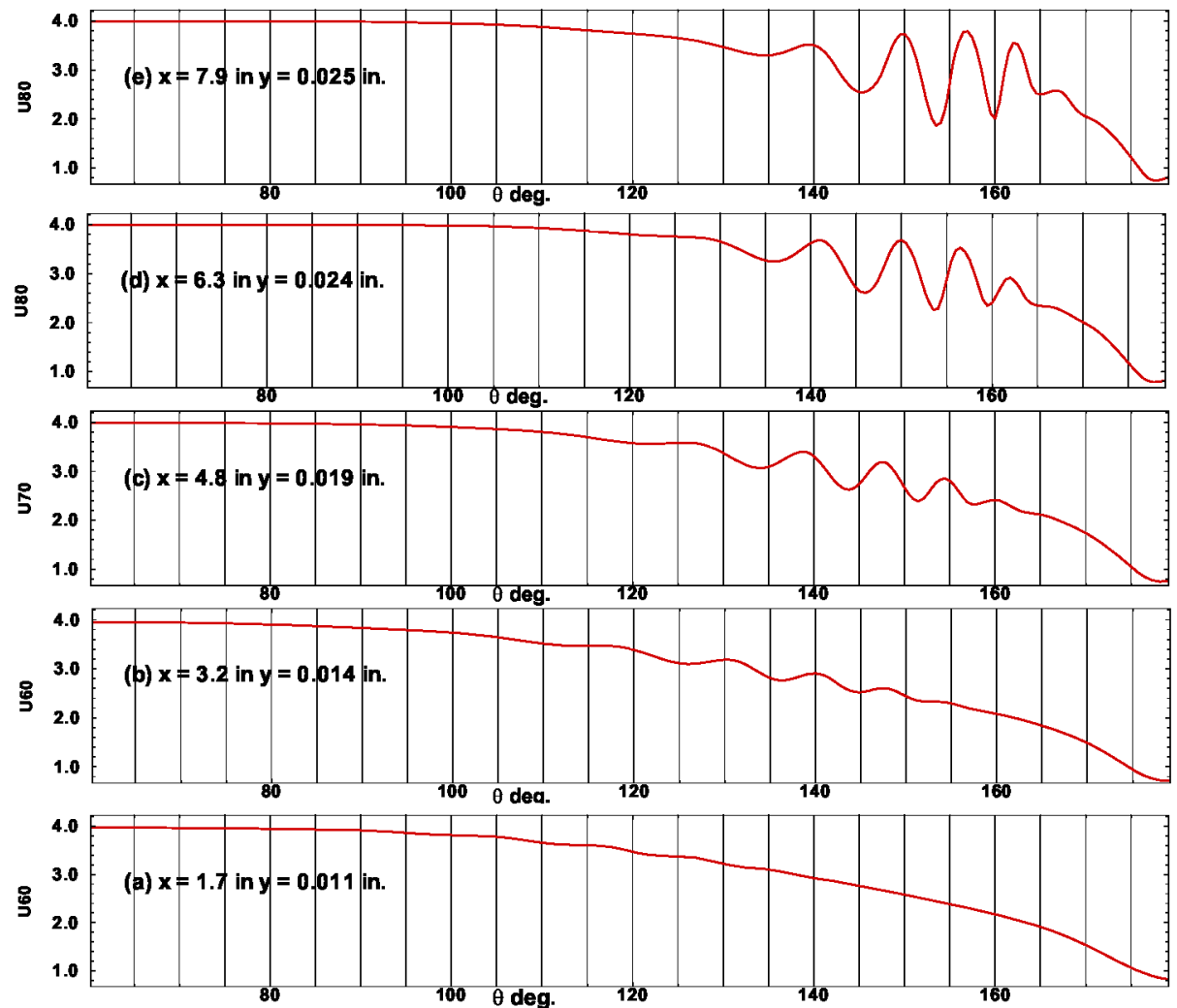

Figure 16. Contours of the axial velocity in the cross planes at different axial locations.

16 of 16

American Institute of Aeronautics and Astronautics AIAA-2009-3555 